\title{
BLUETOOTH BASED OFFICE AUTOMATION AND SECURITY SYSTEM USING RASPBERRY PI AND ANDROID APPLICATION
}

\author{
Dr. Dhiraj Sunehra \\ Department of Electronics \& Communication Engineering \\ Faculty of Engineering \& Technology \\ Jawaharlal Nehru Technological University Hyderabad, Hyderabad
}

\begin{abstract}
An office receptionist is an employee who provides administrative support in the office. The position even though is of much importance, it hinders the use of man power for a much higher productive and useful purpose in the office. This paper deals with design and implementation of an automated office receptionist system using Raspberry $P i$ and an Android application. It not only helps the official to directly interact with the visitor but also to maintain a visitor's record in the e-mail. This eliminates manual assistance and also improves the security. The device can also be used at home, corporate offices, hospitals, etc., for security purpose and administrative purposes. This simple and cost-effective prototype device is designed and when tested it effectively performed the task of office receptionist.
\end{abstract}

Keywords: Raspberry Pi Processor, Office Automation, Bluetooth, Email, Android application

Cite this Article: Dr. Dhiraj Sunehra, Bluetooth Based Office Automation and Security System using Raspberry Pi and Android Application, International Journal of Advanced Research in Engineering and Technology, 10(2), 2019, pp. 449-456.

$\mathrm{http}: / /$ iaeme.com/Home/issue/IJARET?Volume=10\&Issue $=2$

\section{INTRODUCTION}

An office receptionist is an employee who provides administrative support for the smooth and efficient working of an official. The more usual job includes taking the permission to meet the official instead of the visitor and notify them. Obstruct the visitors when the meeting is going on and to keep the record of visitors. This rather results to an improper use of a productive person. An office without automation system will cause interruptions during meeting, discussions or important calls due to visitors. Privacy of official will be disrupted by guests at unexpected time. It will also be difficult to keep track of visitors for security reasons.

A simple device is designed with a novel approach to perform the job of a receptionist as office automation system. This enables them to get involved in other productive activities and to efficiently use man power. The device can also be used in house. This avoids creating disturbances from sales personals, local fundraisers and unwanted interruptions and will be 
secure. In hospitals, this device can be used for notifying the patients waiting outside doctor's cabin about the status of ongoing consultation and for calling the next patient to cabin (where we usually use token system). The major advantages of this prototype are the visitor's identity would be revealed, message interaction over the pressing of a button is provided using Bluetooth. The range of Bluetooth can be extended by using range extenders.

\section{HARDWARE DESCRIPTION}

\subsection{Block Diagram}

Figure 1 shows the block diagram of the automated office receptionist system. The setup consists of Raspberry pi, Router, Bluetooth module, Serial-USB converter, LCD, USB-Camera and Push-button switch. Wireless network consists of Raspberry Pi, Bluetooth module, SerialUSB converter, Push-button, USB-Camera, Bluetooth controller application and LCD. The serial data from the Bluetooth controller application is passed on to the Raspberry pi with the help of Bluetooth module with which status is sent to LCD through wireless communication by Bluetooth module. The Raspberry Pi uploads the status of the official through LCD and the status of the official is controlled through the LCD.

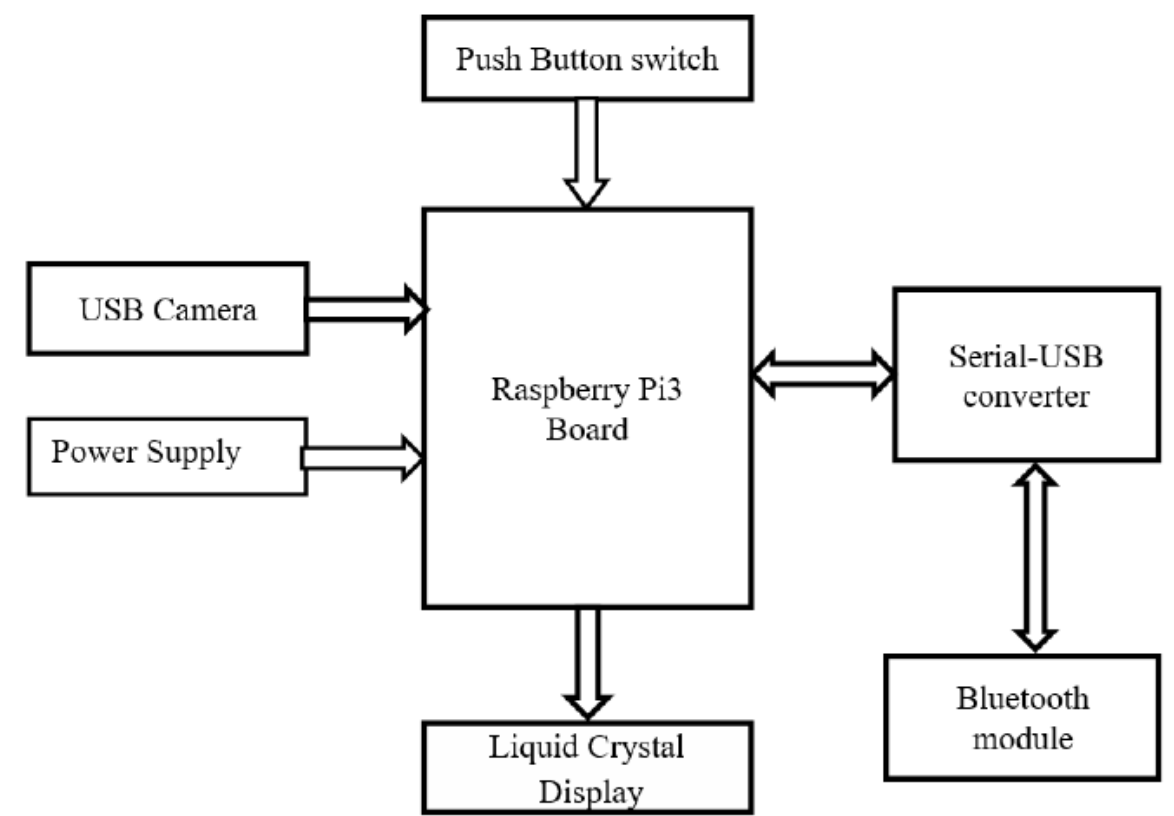

Figure 1 Block Diagram of Automated Office Receptionist System

\subsection{Raspberry Pi Model B+ Board}

Raspberry $\mathrm{Pi}$ is a minicomputer, which is a credit card sized board. It is based on BCM2835 (BCM-Broadcom) system on chip (SoC). Its features include $512 \mathrm{MB}$ of SDRAM, 40 GPIO pins, one Ethernet port, 4 USB ports, HDMI port Audio jack. It has a $700 \mathrm{MHz}$ ARM1176JZFS application processor (Figure 2) [1]. The USB hub can be used to expand the number of ports or to plug the mouse into your keyboard, if it has its own USB port. Ethernet connector allows for wired network access.

A 5V Micro USB power connector is available into which we can plug a compatible power supply. For placing the SD card, a full-sized SD card slot is available. An SD card with an operating system (OS) installed is required for booting the device. The HDMI port provides digital video and audio output. 


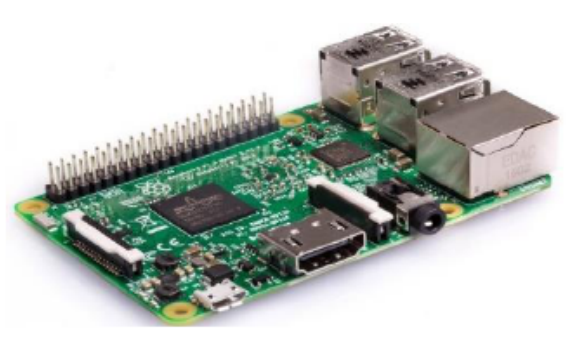

Figure 2 Raspberry Pi Model B+ Board

\subsection{Salient Features of Bluetooth Module}

HC-05 module is an easy to use Bluetooth SPP (Serial Port Protocol) module, designed for transparent wireless serial connection setup [2]. This Bluetooth module can be used in a Master or Slave configuration, making it a great solution for wireless communication (Figure 3). This serial port Bluetooth module is fully qualified Bluetooth V2.0+EDR (Enhanced Data Rate) 3 Mbps modulation with complete $2.4 \mathrm{GHz}$ radio transceiver and baseband. It uses CSR Bluecore 04 - External single chip Bluetooth system with CMOS technology and with AFH (Adaptive Frequency Hopping Feature).

\subsubsection{Hardware Features}

- Typical $-80 \mathrm{dBm}$ sensitivity.

- Up to $+4 \mathrm{dBm}$ RF transmit power.

- 3.3 to $5 \mathrm{~V} \mathrm{I} / \mathrm{O}$.

- PIO (Programmable Input/output) control.

- UART interface with programmable baud rate.

- With integrated antenna.

- With edge connector.

\subsubsection{Software Features}

- Slave default Baud rate: 9600, Data bits: 8, Stop bit:1, Parity: No parity.

- Auto-connect to the last device on power as default.

- Permit pairing device to connect as default.

- Auto-pairing PINCODE:"1234" as default.

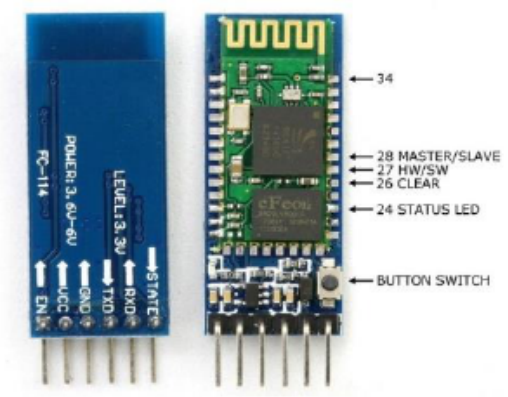

Figure 3 Bluetooth Module

\subsection{Salient Features of Serial USB Converter}

The CP2102 is a highly-integrated USB-to-UART bridge controller providing a simple solution for updating RS-232 designs to USB using a minimum of components and PCB space (Figure 
4). It includes a USB 2.0 full-speed function controller, USB transceiver, oscillator, EEPROM, and asynchronous serial data bus (UART) with full modem control signals in a compact $5 \times 5$ mm MLP-28 package. No other external USB components are required.

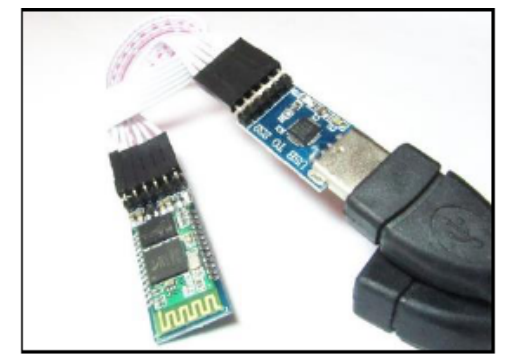

Figure 4 Bluetooth Module - Serial to USB connection

\subsection{Push Button Switch}

A push-button is a simple switch mechanism for controlling some aspect of a machine or a process (Figure 5). Buttons are typically made out of hard material, usually plastic or metal. The surface is usually flat or shaped to accommodate the human finger or hand, so as to be easily depressed or pushed. The "push-button" switch has been utilized in calculators, pushbutton telephones, kitchen appliances, and various other mechanical and electronic devices.

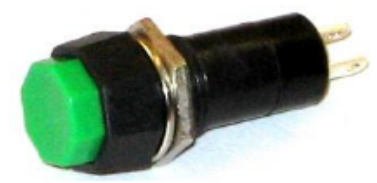

Figure 5 Push Button Switch

\subsection{Liquid Crystal Display}

Liquid Crystal Display (LCD) screen is an electronic display module and finds a wide range of applications. A $16 \times 2$ LCD means it can display 16 characters per line and there are 2 such lines (Figure 6). In this LCD, each character is displayed in 5×7-pixel matrix. This LCD has two registers, viz., command and data. The command register stores the command instructions given to the LCD. A command is an instruction given to LCD to do a predefined task like initializing it, clearing its screen, setting the cursor position, controlling display etc. The data register stores the data to be displayed on the LCD [3]. The data is the ASCII value of the character to be displayed on the LCD.

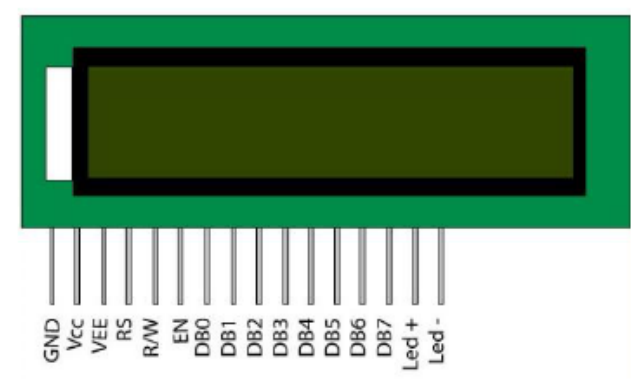

Figure 6 LCD Pin Diagram

\subsection{Web Camera}

A webcam is a video camera that feeds or streams its image in real time to or through a computer to a computer network. When "captured" by the computer, the video stream may be saved, 
viewed or sent on to other networks via systems such as the internet, and emailed as an attachment.

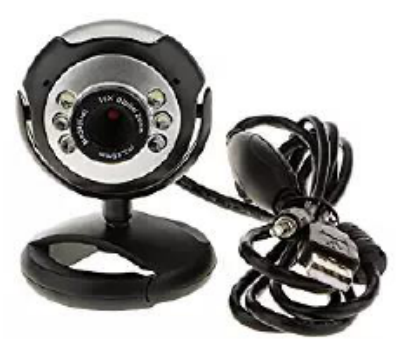

Figure 7 Web Camera

\section{SOFTWARE TOOLS}

ExtraPuTTY is a software tool used to access Raspberry Pi. PuTTY is an open source terminal emulator. It can support various network protocols such as SCP, Telnet, rlogin and raw socket connection [4].

Python language is a high-level script language [5]. Python is portable and it supports on UNIX, Linux and Windows operating system (OS) platforms. Python language fully supports the Raspberry Pi. The written program files are saved with the extension of .py.

\section{FLOWCHART}

Figure 8 shows the flowchart of the sequence of events that occur in the automated office receptionist system.

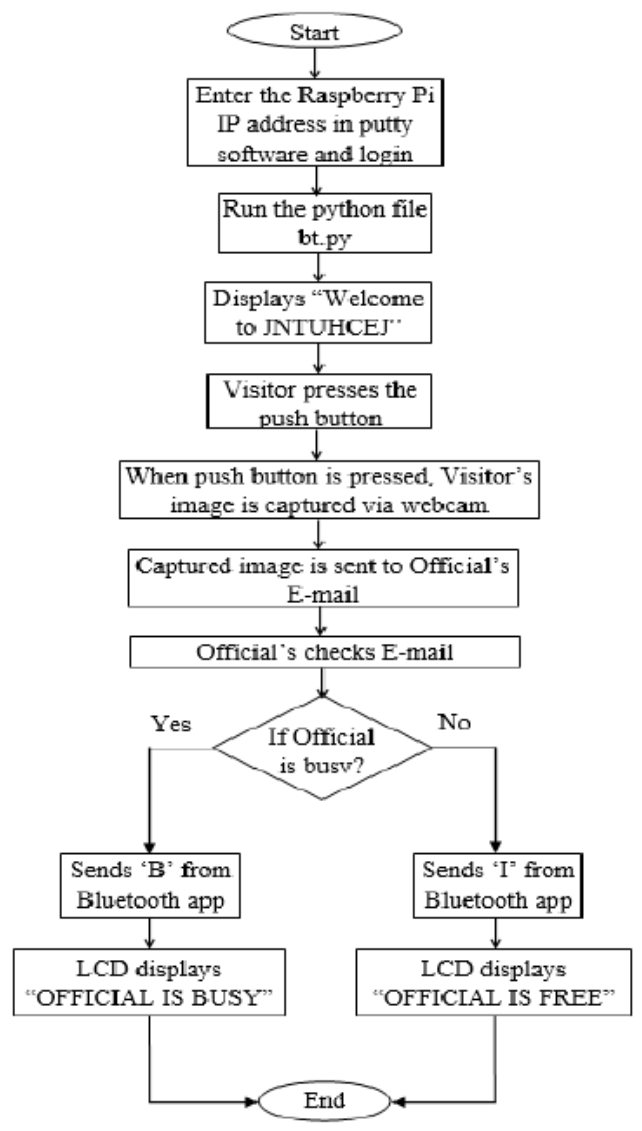

Figure 8 Flowchart of Automated Office Receptionist system 


\section{EXPERIMENTAL SETUP}

Figure 9 shows the experimental setup of automated office receptionist system. The setup consists of Raspberry Pi, router, Bluetooth module, Serial-USB converter, LCD, USB-Camera and Push-button. The serial data from the Bluetooth controller application is passed on to the Raspberry Pi with the help of Bluetooth module with which status is sent to LCD through wireless communication by Bluetooth module. The Raspberry Pi uploads the status of the official on the LCD.

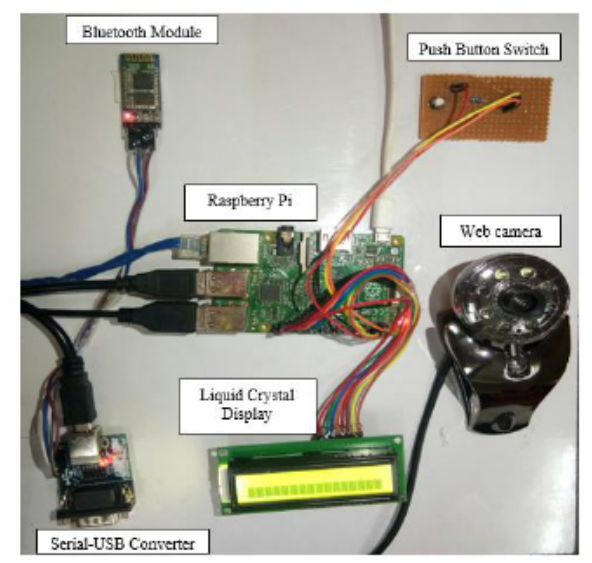

Figure 9 Experimental Setup of the System

\section{EXPERIMENTAL RESULTS}

\subsection{Accessing Raspberry Pi through PuTTY Software}

The initialization of PuTTY software for accessing Raspberry Pi is done using IP address of the Pi (Figure 10).

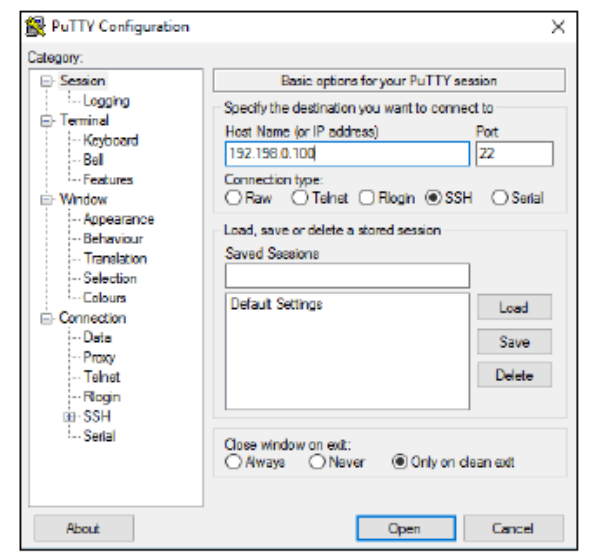

Figure 10 PuTTY Window

Login to Raspberry Pi contains login id as pi and password (Figure 11). After logging in, redirect to the folder where files are stored in Raspberry Pi and open the files by using $l s$ command in Linux. After finding the file, to execute it use nano command nano $<$ file_name $>$.

Figure 11 Logging in to Raspberry Pi 
On executing or running python file to enable the wireless office automation and security system, the data on LCD is refreshed automatically (Figure 12). The status of the official i.e., whether the official is frees to meet or not is displayed on the LCD.

\subsection{When Push-button is pressed}

pilraspberrypi: /ofc \$
pilraspberrypi: /ofc \$ python LCD_TestPrint.p
pilraspberrypi: /ofc \$ python bt.py
Welcome to JNTUHCEJ

Figure 12 Running Python file to capture the image of the visitor

When the push button is pressed, the USB-Camera captures the image of the visitor and it is sent to the official via E-mail (Figure 13). It also acts as a security from preventing the invaders entering the office premises.

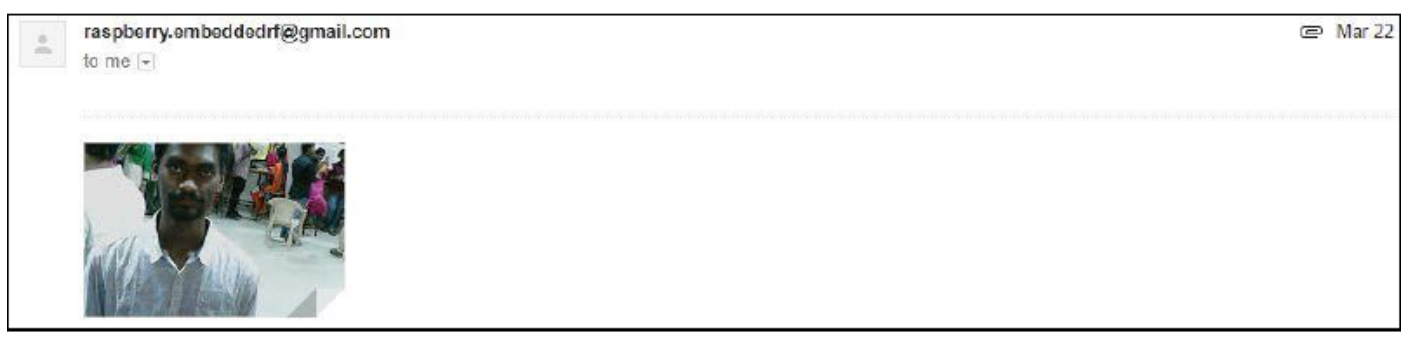

Figure 13 Captured image of the visitor which is sent to the official's email address

\subsubsection{Case 1: Official is free}

The official gives serial input 'I' through the application if he is free (Figure 14). The LCD then displays as "Official is free" (Figure 15).

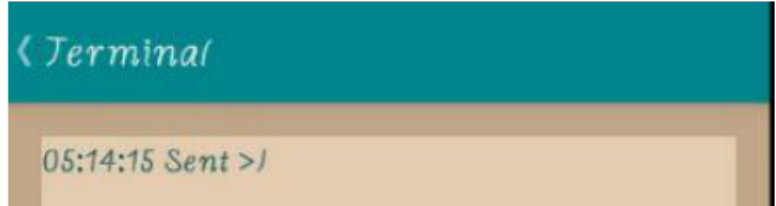

Figure 14 Serial input ' $I$ ' is given through application by the official if he is free

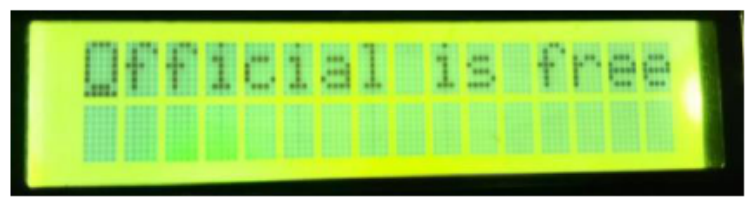

Figure 15 Status "Official is free" is displayed on LCD

\subsubsection{Case 2: Official is busy}

The official gives serial input ' $B$ ' through the application if he is busy (Figure 16). The LCD then displays as "Official is busy" (Figure 17).

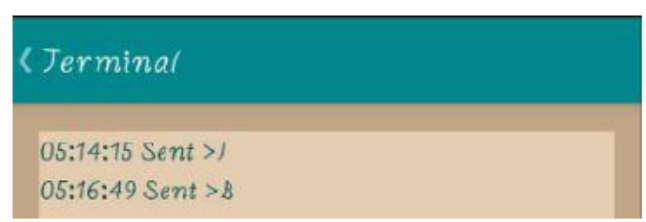

Figure 16 Serial input ' $\mathrm{B}$ ' is given through application by the official if he is busy 


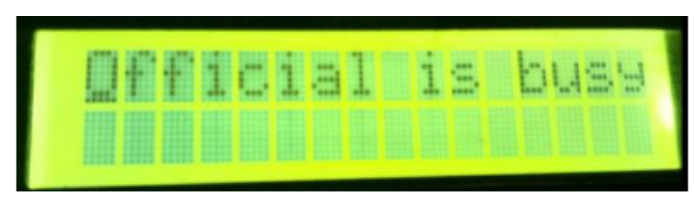

Figure 17 Status "Official is busy" is displayed on LCD

\section{CONCLUSIONS}

A simple and cost-effective prototype has been designed and implemented which performs the task of an Office receptionist. It allows the official to directly interact with the visitor and helps in maintaining an Image-time record in E-mail for security purpose. This can also be used in for residential applications, corporate offices, hospitals, etc. The results have shown that one can easily operate the device effectively. The work can be extended by modifying the system to automatically respond to the visitor by integrating an ultrasonic distance sensor. Also a web server can be included, so that the official can interact with the visitor from anywhere in the world.

\section{REFERENCES}

[1] https://www.raspberrypi.org/

[2] https://www.mynics.in/product/bluetooth-module-hc-05/

[3] https://circuitdigest.com

[4] https://en.wikipedia.org/wiki/PuTTY.

[5] Richard L. Halterman, "Learning to Program with Python", 2011 (www.cs.uky.edu/).

[6] Anilkumar B and KRJ Srikanth, Design and Development of Real Time Paper Currency Recognition System of Demonetization New Indian Notes by Using Raspberry Pi for Visually Challenged, International Journal of Mechanical Engineering and Technology, 9(3), 2018, pp. 884-891.

[7] Bhagya Maybel J and A. Umamakeswari, Hardware Implementation of Secure Image Transmission in Raspberry PI, International Journal of Mechanical Engineering and Technology 9(2), 2018, pp. 670-678.

[8] Trideep Singha Roy, Soumalya Ghosh, Rimpi Datta, Arpita Santra, IoT Based Home Automation Using Raspberry PI, International Journal of Computer Engineering and Technology 10(3), 2019, pp. 70-74

[9] Dr.Raju Anitha, S.Harsha Vardhan, Ch.Akhil and G.Sona, An Automated SMS-Update System through IOT using Raspberry Pi, International Journal of Mechanical Engineering and Technology 9(1), 2018, pp. 118-124.

[10] Dr. Dhiraj Sunehra, Biometric Based Attendance Registration and Consolidation System Using Raspberry PI3 and Amazon Web Server, International Journal of Advanced Research in Engineering and Technology, 10 (2), 2019, pp 34-43. 